\title{
The impact of pore size distribution data presentation format on pore structure interpretation of shales
}

\author{
Kouqi Liu, Mehdi Ostadhassan ${ }^{\oplus} *$ \\ Department of Petroleum Engineering, University of North Dakota, Grand Forks, ND 58202, USA
}

(Received Feburary 20, 2019; revised March 21, 2019; accepted March 22, 2019; available online March 29, 2019)

Citation:

Liu, K., Ostadhassan, M. The impact of pore size distribution data presentation format on pore structure interpretation of shales. Advances in Geo-Energy

Research, 2019, 3(2): 187-197, doi:

10.26804/ager.2019.02.08.

Corresponding author:

*E-mail: mehdi.ostadhassan@und.edu

Keywords:

Pore size distribution

different presentations

multifractal

gas adsorption

mercury intrusion

\begin{abstract}
:
A deeper understanding of pore structures in unconventional shale plays can lead to a better evaluation of storage and transport capacity in these complicated rock types. This task is usually done through pore size distribution (PSD) analysis. In this study, $\mathrm{N}_{2}$ adsorption and high-pressure mercury intrusion porosimetry (MIP) were employed to investigate several shale samples. Three different mathematical forms of PSD data presentation: Incremental pore volume versus diameter $(D V)$, differential pore volume versus diameter $(D V / D d)$ and the $\log$ differential pore volume versus diameter $(D V / D \log d)$, were used to analyze pore structures from these two different methods. The comparison of the results showed that each form of PSD data presentation could demonstrate various types of important pore information. The $D V$ curve is significantly dependent on the experimental data points' spacing while the other two are not affected. The $D V / D d$ curve would incite the existence of smaller pore ranges while the $D V / D \log d$ would embolden larger pore ranges. Additionally, multifractal analysis from each data presentation style illustrated that the heterogeneity index of PSD calculated from the $D V / D d$ curve is much significant than the one obtained from the $D V / D \log d$ curve. $D V / D d$ is more appropriate to be used for characterizing PSD data from $\mathrm{N}_{2}$ adsorption while $D V / D \log d$ is preferred when MIP data is collected from larger pores.
\end{abstract}

\section{Introduction}

As a typical unconventional reservoir, nowadays shales contribute to a large portion of the total oil/gas production in the world. The pore spaces in the shale rocks are the place where hydrocarbons are accumulated, while the size of these pores could affect the transporting behavior of the rock medium (Liu and Peyman, 2017). Thus, accurately characterizing the pore size distribution of the shale rocks is essential. During the past decade, many researchers have investigated the pore structures of different shale plays in the world (Yang et al., 2015; Wang et al., 2016, 2019; Kumar et al., 2019). In this regard, fluid invasion methods such as the gas adsorption and high-pressure mercury intrusion porosimetry (MIP) are widely applied in characterizing the pore structures of shale rocks (Bustin et al., 2008; Clarkson et al., 2012, 2013; Gao and $\mathrm{Hu}, 2013$; Mastalerz et al., 2013; Tang et al., 2015; Han et al., 2016; Liu et al., 2017, 2019a; Zou et al., 2017; Yuan et al., 2018).

It is known that in nitrogen adsorption, as the relative pressure (the ratio of the gas vapor pressure in the system and the saturation pressure of nitrogen) increases, the adsorption quantity will increase (Thommes et al., 2015). This will enable the estimation of pore volume based on the assumption of the existence of certain pore shapes within the sample. In highpressure mercury intrusion, Washburn equation is commonly employed to quantify the pore sizes (Washburn, 1921). The pore size distribution derived from these two latest methods is usually expressed in the form of a pore volume distribution as a function of the pore diameter/radius. Incremental pore volume versus diameter $(D V)$, differential pore volume versus diameter $(D V / D d)$ and the log differential pore volume versus diameter $(D V / D \log d)$ are three main presentations that researchers use to display pore size distributions. These three presentations of the pore size can be calculated through the following equations (Meyer and Klobes, 1999):

$$
\begin{gathered}
D V=V_{i+1}-V_{i} \\
\frac{D V}{D d}=\frac{V_{i+1}-V_{i}}{d_{i+1}-d_{i}} \\
\frac{D V}{D \log d}=\frac{V_{i+1}-V_{i}}{\log d_{i+1}-\log d_{i}}
\end{gathered}
$$


Table 1. Mineral compositions of the samples tested for the $\mathrm{N}_{2}$ gas adsorption.

\begin{tabular}{lllllll}
\hline Samples & $\begin{array}{l}\text { Quartz } \\
(\text { wt. \%) }\end{array}$ & $\begin{array}{l}\text { Feldspar } \\
(\text { wt. \%) }\end{array}$ & $\begin{array}{l}\text { Carbonate } \\
(\text { wt. \%) }\end{array}$ & $\begin{array}{l}\text { Pyrite } \\
(\text { wt. \%) }\end{array}$ & $\begin{array}{l}\text { Clay } \\
\text { (wt. \%) }\end{array}$ & $\begin{array}{l}\text { TOC } \\
\text { (wt. \%) }\end{array}$ \\
\hline$\# 1$ & 60.2 & 8.1 & - & 2.4 & 20.8 & 13.1 \\
$\# 2$ & 46.1 & 1.3 & 2.1 & 15 & 32.3 & 17.7 \\
$\# 3$ & 57.8 & 10.7 & 3.8 & 3.9 & 20.3 & 5.4 \\
$\# 4$ & 55.1 & 1.2 & 22.1 & 2.2 & 2.4 & 5.9 \\
$\# 5$ & 15.1 & 0.2 & 66.7 & 5.6 & 10.1 & 6.8 \\
$\# 6$ & 30.6 & 2.0 & 46.6 & 1.7 & 14.9 & 4.8 \\
\hline
\end{tabular}

where $V_{i}$ is the cumulative pore volume at the pressure point $\left(P_{i}\right)$ and $V_{i+1}$ is the cumulative pore volume at a higherpressure $\left(P_{i+1}\right) . d_{i+1}$ refers to the pore diameter where the cumulative pore volume is $V_{i+1}$ and $d_{i}$ donates the pore diameter where the cumulative pore volume is $V_{i}$.

Choosing the most appropriate form of presentation of pore size distribution (PSD) could affect the pore structure interpretations. This discrepancy in interpretation of the results, originates from different mathematical concepts that these methods rely on. However, to the authors' knowledge, very few studies are available that compares these different presentations and the outcomes, specifically related to the pore structure characterization of shales. In certain methods, such as $\mathrm{N}_{2}$ adsorption, some researchers utilized the $D V$ (Cao et al., 2015) while other researchers employed $D V / D d$ (Kuila, 2013) or $D V / D \log d$ (Clarkson et al., 2013; Liu et al., 2017). However, the question remains how each presentations method would interpret the data and the superiority of one over the other to better characterize the pore structures of the shale with $\mathrm{N}_{2}$ adsorption or mercury intrusion, in particular.

In this paper, we analyzed pore structure data and compared different forms of presentations of the PSD data from shale rocks that were acquired by $\mathrm{N}_{2}$ adsorption and highpressure mercury intrusion methods. The main goal of this paper is to add more knowledge to our understanding from the pore structure of shale rocks through in-depth data analysis and comparison by answering the following questions: 1) What is the difference between these different common forms of data presentations? Also, 2) which presentation is preferred for the $\mathrm{N}_{2}$ adsorption and which one is more suited towards highpressure mercury intrusion.

\section{Case study and discussions}

\section{$2.1 N_{2}$ adsorption}

6 shale samples were crushed into powders and then degassed for more than 8 hours at $110{ }^{\circ} \mathrm{C}$ (Liu et al., 2019b). It is well known that grain sizes could affect data analysis and the results (Hazra et al., 2018), thus, in order to limit grain size effect, in this study, samples were prepared to have grain sizes less than $250 \mu \mathrm{m}$. Then $\mathrm{N}_{2}$ adsorption experiment was performed with the Micromeritics ${ }^{\circledR}$ Tristar II apparatus at 77 $\mathrm{K}$. The gas adsorption volume was measured over the relative equilibrium adsorption pressure $\left(P / P_{0}\right)$ ranging from 0.01 to
0.99 . After that, the pore size distributions of the samples were calculated using the density functional theory (Do and Do, 2003).

The major mineral compositions and the total organic carbon (TOC) content of the samples that were tested by $\mathrm{N}_{2}$ adsorption can be seen in Table 1. The results showed samples having different mineral compositions and TOC content. Quartz and clay are the major minerals in Sample \#1 to Sample \#4 while the dominant mineral in Sample \#5 and Sample \#6 were carbonate minerals. Sample \#2 has the largest TOC content (17.7 wt. \%) while Sample \#6 has the smallest TOC content (4.8 wt. \%).

The simplest way to display pore size distribution is to plot the incremental pore volume versus the pore diameter. Fig. 1 shows the incremental pore volume curves of two representative samples (Sample \#1 and Sample \#2). Both curves exhibit the multimodal peaks, depicting the complexity of the pore structures. The largest peak exists between the pore size intervals from $20 \mathrm{~nm}$ to $30 \mathrm{~nm}$ for both samples, indicating that the pores with sizes from $20-30 \mathrm{~nm}$ contribute the most to the porosity of the samples when the PSD data is presented in this specific style. $D V$ curve is the true pore volume distribution that is a function of pore diameter. However, the peak values from this distribution are dependent on the experiment spacing data points since more pore volume will be accumulated by summation in a larger pore radius interval than in the smaller ones (Meyer and Klobes, 1999). The limit of the pore size that can be measured by $\mathrm{N}_{2}$ adsorption is less than $200 \mathrm{~nm}$. In this experiment, relative pressure was increased from 0 to 0.99 linearly, while, based on Kelvin's equation, the relationship between the pore diameter and the relative pressure is non-linear. The incremental pore diameter between the two neighboring experimental data point is increasing as the pore size increases. In order to avoid the effect that spacing between data points would have on the pore size distribution, two other presentations of pore size distribution: Differential pore volume distribution and log differential pore volume distribution are developed.

The blue curve in Figs. 2(a) and 2(b) shows the differential pore volume distribution of Sample \#1 and Sample \#2, respectively. The incremental pore volume can be calculated from the area under the blue curve. Similarly, the $D V / D d$ curve also demonstrates the multimodal characteristics which was observed earlier in the sample. Comparing these two curves 


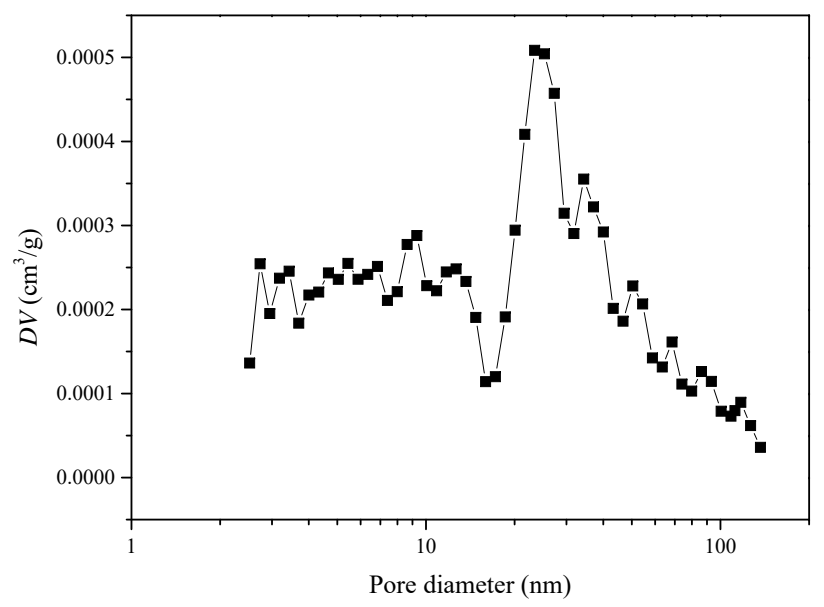

(a) Sample \#1

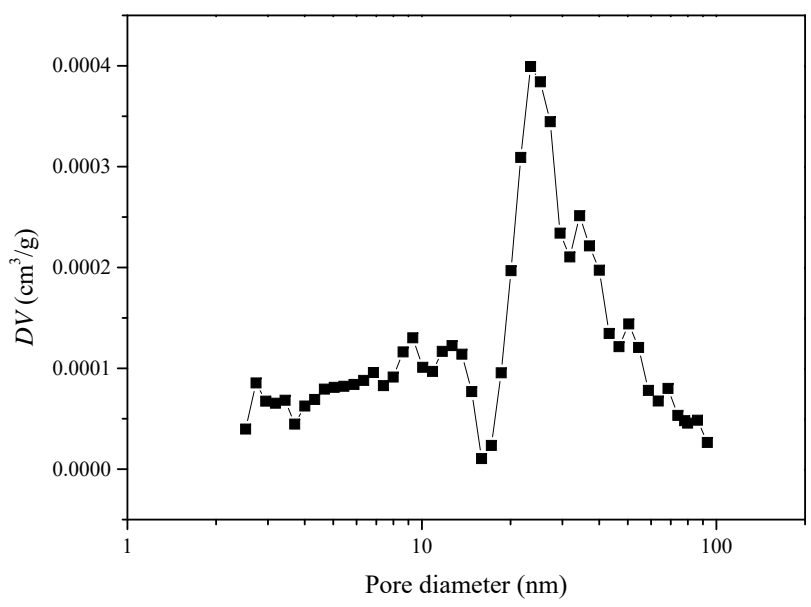

(b) Sample \#2

Fig. 1. Examples of the incremental pore volume versus the pore diameter.

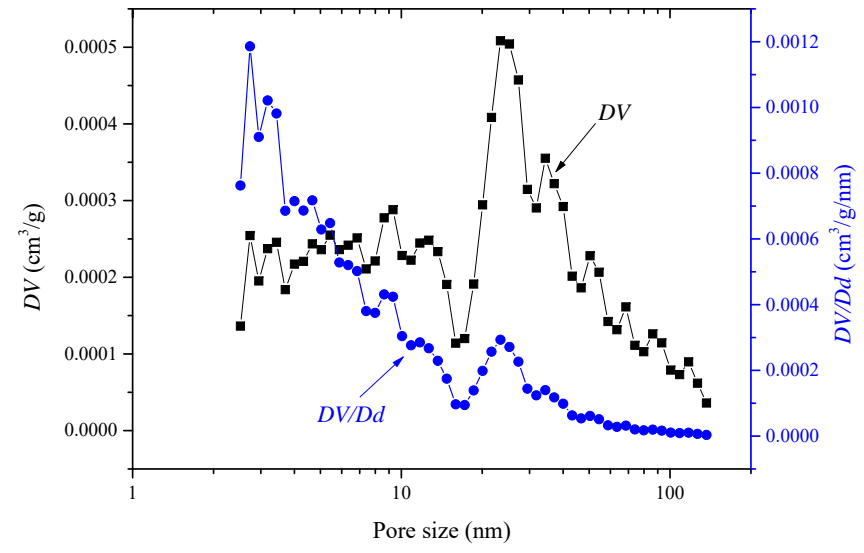

(a) Sample \#1

Fig. 2. Comparison of the $D V$ curve (black) and the $D V / D d$ curve (blue).

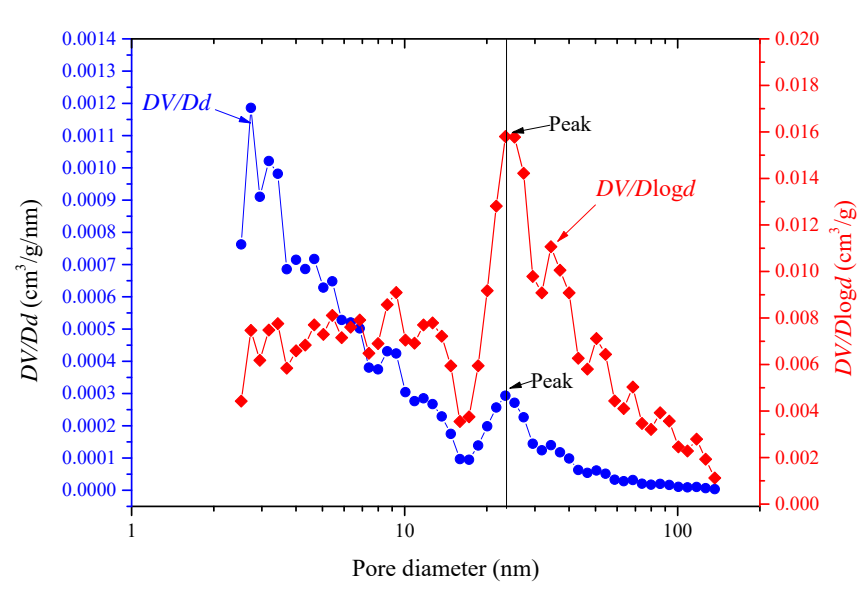

(a) Sample \#1

Fig. 3. Comparison of the $D V / D d$ curve (blue) and the $D V / D \log d$ curve (red).

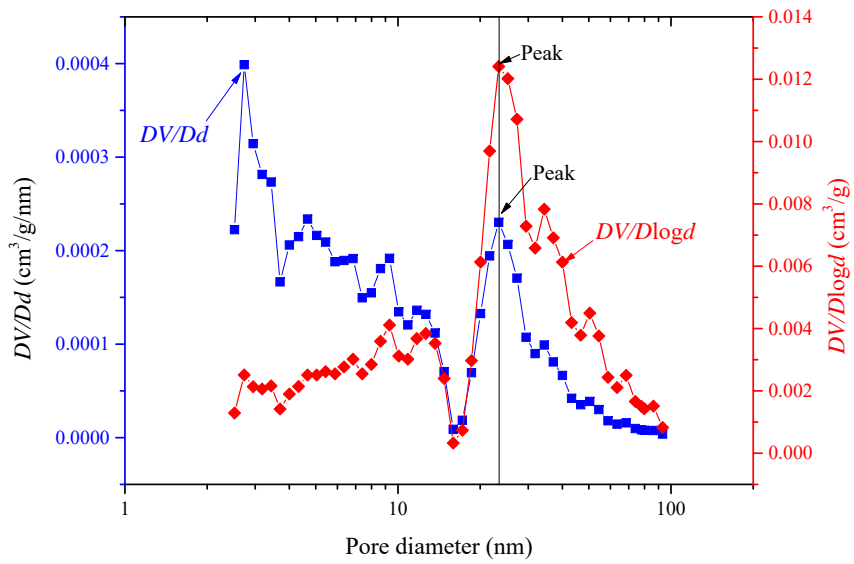

(b) Sample \#2

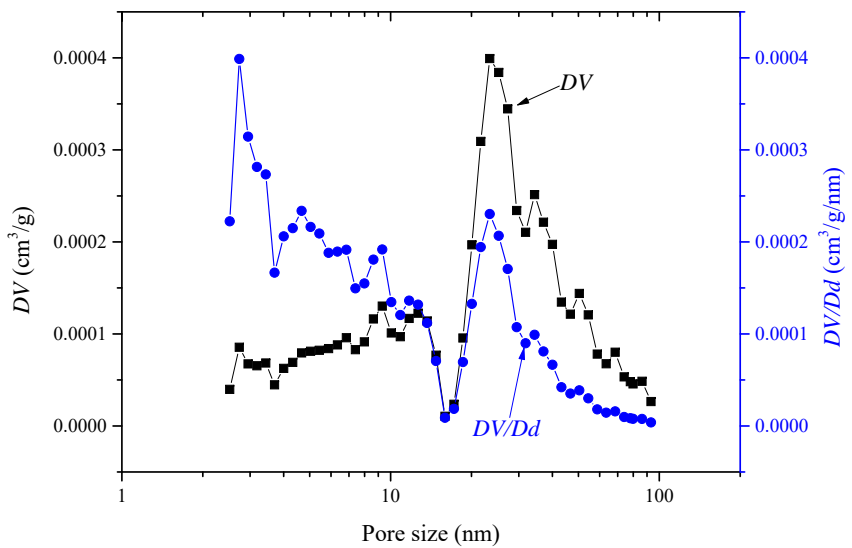

(b) Sample \#2 


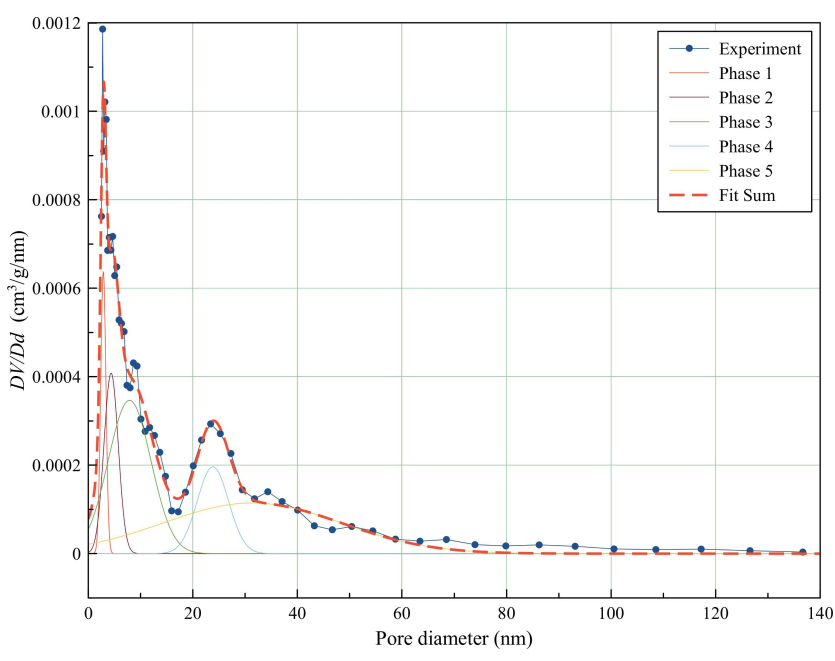

(a) $D V / D d$

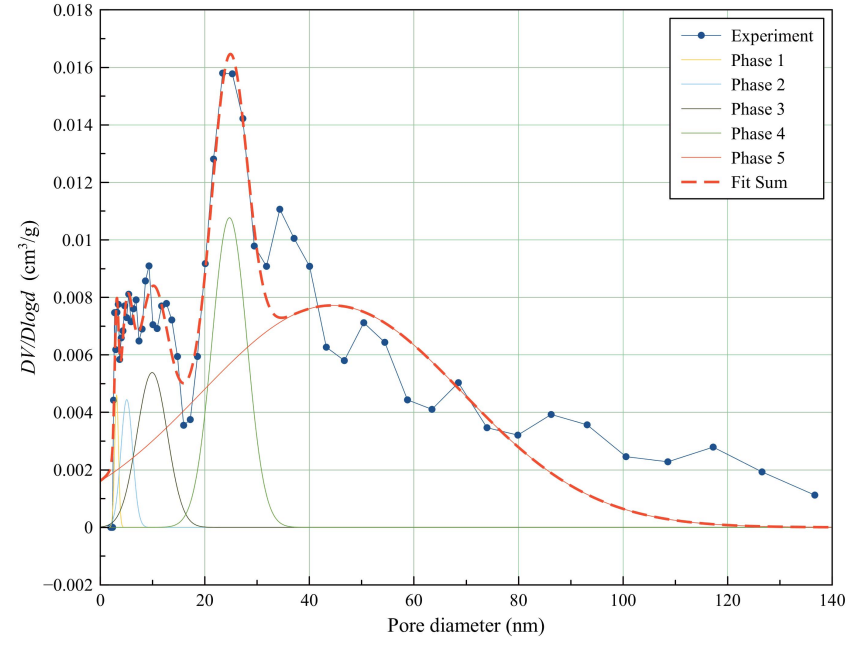

(b) $D V / D \log d$

Fig. 4. Deconvolution results of the pore size distributions of Sample \#1 in two different presentations.

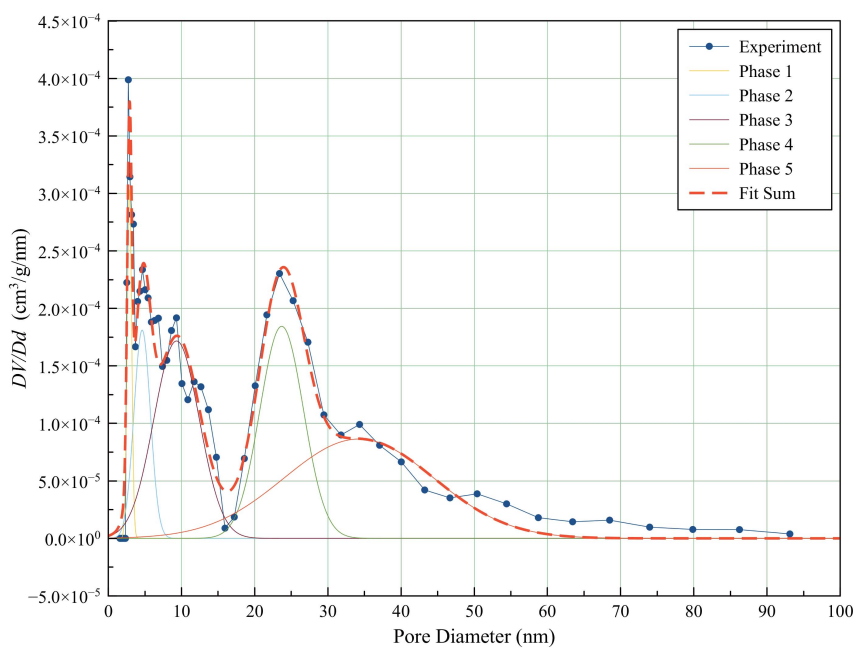

(a) $D V / D d$

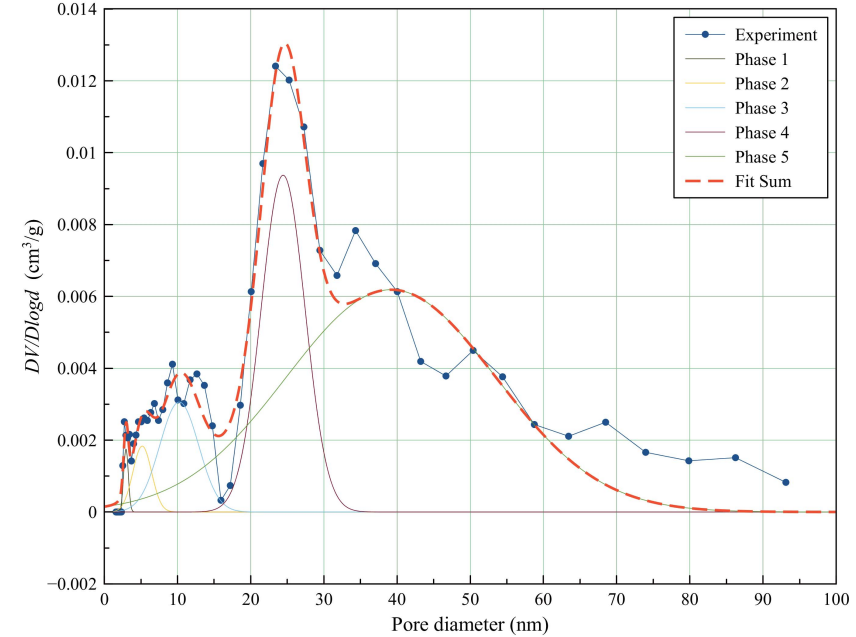

(b) $D V / D \log d$

Fig. 5. Deconvolution results of the pore size distributions of Sample \#2 in two different presentations.

Fig. 2, the major peak representing dominant pore sizes is found around 20 to $30 \mathrm{~nm}$ by the $D V$ curve, while the major peak of the $D V / D d$ curve (blue) is observed around 2-4 $\mathrm{nm}$ as the dominant pore size.

The log differential pore volume distribution curve $(D V / D \log d)$ and the differential pore volume distribution curve $(D V / D d)$ of Sample \#1 and Sample \#2 can be seen in Fig. 3. The largest peak of the $D V / D \log d$ curve is around 20-30 nm which is closer to the location of the largest peak in the $D V$ curve (Fig. 3). The location of the peaks can be derived from the deconvolution method. The details of this procedure can be seen in the appendix. The deconvolution of the $D V / D d$ curve and the $D V / D \log d$ curve of Sample \#1 and Sample \#2 can be found in Figs. 4 and . 5, respectively. Considering Sample \#1, both $D V / D d$ and $D V / D \log d$ curves can be deconvoluted into 5 different phases. The deconvolution summary results from all of the samples based on $\mathrm{N}_{2}$ adsorption data can be seen in Table 2. Considering Sample \#1 for instance, the peaks of the pore size range of 10-40 nm can be seen in both $D V / D d$ and the $D V / D \log d$ curves, meaning that these peaks are not affected with the style of PSD data presentations. On the contrary, considering Sample \#3, comparing $D V / D \log d$ and $D V / D d$ curves, the latest, shows the presence of additional peaks in the smaller pore radii range while $D V / D \log d$ provides us with peaks in the larger pore size ranges. Based on the results from this step, it can be said that $D V / D d$ curve can reflect more information about the pores in the smaller radius range while $D V / D \log d$ in the larger pore radius range.

Pore size distribution data can be used to quantify the heterogeneity of pore structures. In this section, multifractal analysis is employed to analyze and compare the heterogeneity index of pore size distributions from these two different presentations $(D V / D d$ and $D V / D \log d)$. The detail of multi- 
Table 2. The deconvolution results of two different presentations from $\mathrm{N}_{2}$ adsorption.

\begin{tabular}{|c|c|c|c|c|c|c|c|c|c|c|c|}
\hline \multirow{2}{*}{ Samples } & & \multicolumn{2}{|c|}{ Phase 1} & \multicolumn{2}{|c|}{ Phase 2} & \multicolumn{2}{|c|}{ Phase 3} & \multicolumn{2}{|c|}{ Phase 4} & \multicolumn{2}{|c|}{ Phase 5} \\
\hline & & $\begin{array}{l}\text { Mean } \\
(\mathrm{nm})\end{array}$ & $\begin{array}{l}\mathrm{Sd} \\
(\mathrm{nm})\end{array}$ & $\begin{array}{l}\text { Mean } \\
(\mathrm{nm})\end{array}$ & $\begin{array}{l}\mathrm{Sd} \\
(\mathrm{nm})\end{array}$ & $\begin{array}{l}\text { Mean } \\
(\mathrm{nm})\end{array}$ & $\begin{array}{l}\mathrm{Sd} \\
(\mathrm{nm})\end{array}$ & $\begin{array}{l}\text { Mean } \\
(\mathrm{nm})\end{array}$ & $\begin{array}{l}\mathrm{Sd} \\
(\mathrm{nm})\end{array}$ & $\begin{array}{l}\text { Mean } \\
(\mathrm{nm})\end{array}$ & $\begin{array}{l}\mathrm{Sd} \\
(\mathrm{nm})\end{array}$ \\
\hline \multirow{2}{*}{ \#1 } & 1 & 2.78 & 0.06 & 4.36 & 0.68 & 7.89 & 3.00 & 23.83 & 0.87 & 31.37 & 20.07 \\
\hline & 2 & 3.11 & 0.13 & 5.06 & 0.43 & 9.92 & 0.94 & 24.71 & 0.42 & 44.31 & 4.31 \\
\hline \multirow{2}{*}{ \#2 } & 1 & 2.89 & 0.03 & 4.63 & 0.18 & 9.33 & 0.70 & 23.69 & 0.51 & 34.24 & 6.69 \\
\hline & 2 & 2.96 & 0.16 & 5.18 & 0.68 & 10.23 & 0.89 & 24.43 & 0.27 & 39.18 & 2.17 \\
\hline \multirow{2}{*}{ \#3 } & 1 & 2.73 & 0.04 & 3.82 & 0.39 & 6.04 & 0.71 & 10.15 & 2.87 & 21.36 & 6.77 \\
\hline & 2 & 2.97 & 0.04 & 5.83 & 0.22 & 10.72 & 1.71 & 23.95 & 2.87 & 73.43 & 16.83 \\
\hline \multirow[t]{2}{*}{ \#4 } & 1 & 8.27 & 0.64 & 12.42 & 1.71 & 19.87 & 2.25 & 37.62 & 0.45 & 52.19 & 4.22 \\
\hline & 2 & 9.50 & 1.87 & 15.82 & 4.76 & 22.07 & 3.58 & 38.54 & 0.46 & 63.85 & 2.49 \\
\hline \multirow{2}{*}{$\# 5$} & 1 & 3.21 & 0.03 & 4.73 & 0.18 & 9.97 & 0.36 & 24.37 & 0.29 & 35.81 & 3.34 \\
\hline & 2 & 3.28 & 0.29 & 8.85 & 2.04 & 12.75 & 0.66 & 25.17 & 0.31 & 40.78 & 1.91 \\
\hline \multirow[t]{2}{*}{$\# 6$} & 1 & 3.04 & 0.05 & 4.27 & 0.31 & 8.48 & 1.01 & 24.03 & 1.16 & 34.32 & 9.69 \\
\hline & 2 & 3.13 & 0.14 & 4.65 & 0.63 & 9.57 & 1.14 & 25.00 & 0.41 & 45.46 & 3.40 \\
\hline
\end{tabular}

Note: Sd means the standard deviation, 1 refers to the $D V / D d$ curve while 2 denotes the $D V / D \log d$ curve.

Table 3. Summary of multifractal analysis from these two different presentations obtained from $\mathrm{N}_{2}$ adsorption.

\begin{tabular}{lllllllll}
\hline \multirow{2}{*}{ Samples } & \multicolumn{3}{c}{$D V / D d$} & \multicolumn{4}{c}{$D V / D \log d$} \\
\cline { 2 - 9 }$\#$ \#1 & $\alpha_{-10}$ & $\alpha_{0}$ & $\alpha_{10}$ & $\alpha_{-10}-\alpha_{10}$ & $\alpha_{-10}$ & $\alpha_{0}$ & $\alpha_{10}$ & $\alpha_{-10}-\alpha_{10}$ \\
$\# 2$ & 2.4862 & 1.6404 & 0.1419 & 2.3443 & 1.8259 & 1.2233 & 0.4317 & 1.3942 \\
$\# 3$ & 2.1494 & 1.4358 & 0.2221 & 1.9273 & 1.6453 & 1.1504 & 0.5294 & 1.1159 \\
$\# 4$ & 2.2335 & 1.5762 & 0.1871 & 2.0464 & 1.5618 & 1.1761 & 0.4664 & 1.0954 \\
$\# 5$ & 1.7134 & 1.2440 & 0.4992 & 1.2142 & 1.2652 & 1.0617 & 0.6761 & 0.5891 \\
$\# 6$ & 1.7221 & 1.3150 & 0.3350 & 1.3871 & 1.3357 & 1.1095 & 0.6903 & 0.6454 \\
\hline
\end{tabular}

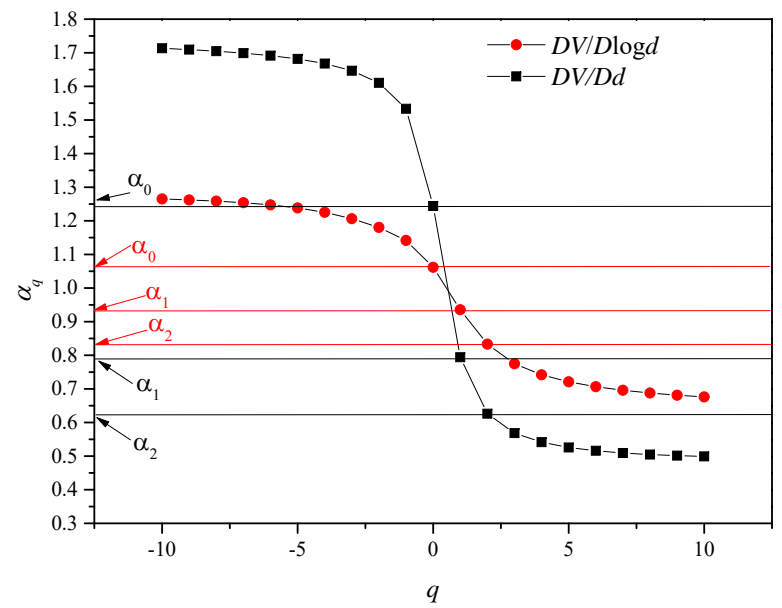

Fig. 6. The relationship between $\alpha_{q}$ and $q$ in Sample \#4. fractal analysis procedure can be found in Liu et al. (2018). Heterogeneity index can be defined as the difference between $\alpha_{-10}$ and $\alpha_{10}$, where $\alpha_{-10}$ and $\alpha_{10}$ are singularity exponents when the fractal exponent $q$ is equal to -10 and 10 , respectively. The correlations between $\alpha_{q}$ and $q$ in Sample \#4 can be seen in Fig. 6. $\alpha_{0}, \alpha_{1}$, and $\alpha_{2}$ refer to the singularity exponents as $q$ equals to 0,1 and 2, respectively. $\alpha_{0}, \alpha_{1}$ and $\alpha_{2}$ of both curves follows this order: $\alpha_{0}>\alpha_{1}>\alpha_{2}$ (Fig. 6), which infers that pore size distribution of these two different presentations exhibits the multifractal behavior (Ferreiro et al., 2010; Liu et al., 2018).

The results from the multifractal analysis of all samples are summarized in Table 3 . The results depicted that for the same sample, the multifractal parameters, $\alpha_{-10}$ and $\alpha_{0}$ that are obtained from the $D V / D d$ curve are larger than the same 
Table 4. Mineral compositions of the samples for the mercury intrusion test.

\begin{tabular}{lllllll}
\hline Samples & $\begin{array}{l}\text { Quartz } \\
\text { (wt. \%) }\end{array}$ & $\begin{array}{l}\text { Pyrite } \\
\text { (wt. \%) }\end{array}$ & $\begin{array}{l}\text { Feldspar } \\
\text { (wt. \%) }\end{array}$ & $\begin{array}{l}\text { Dolomite } \\
\text { (wt. \%) }\end{array}$ & $\begin{array}{l}\text { Clay } \\
\text { (wt. \%) }\end{array}$ & $\begin{array}{l}\text { TOC } \\
\text { (wt. \%) }\end{array}$ \\
\hline$\# 7$ & 70.3 & 3.2 & 7.7 & 0.0 & 18.8 & 14.2 \\
$\# 8$ & 54.3 & 8.1 & 0.0 & 8.8 & 28.6 & 20.2 \\
$\# 9$ & 66.9 & 2.4 & 14.4 & 0.0 & 16.2 & 11.1 \\
$\# 10$ & 70.0 & 2.4 & 5.5 & 0.0 & 22.2 & 12.2 \\
$\# 11$ & 36.1 & 8.5 & 3.4 & 0.0 & 52.0 & 14.9 \\
\hline
\end{tabular}

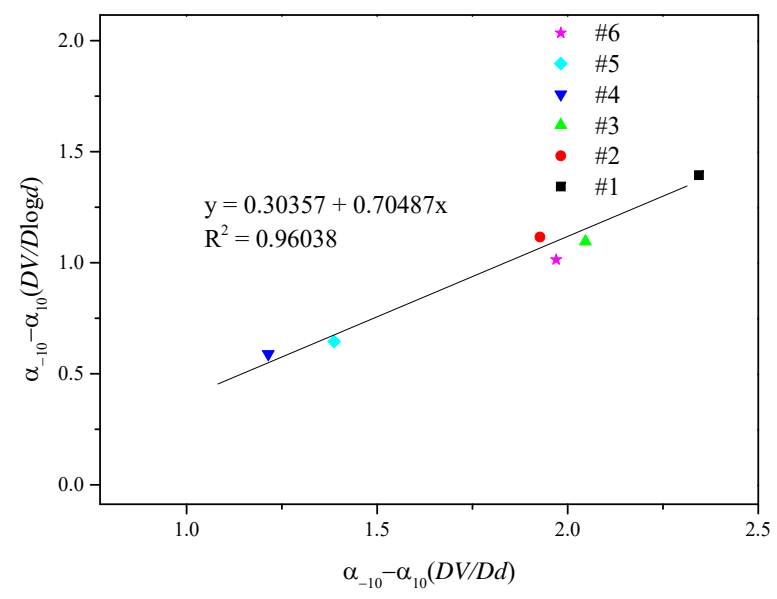

Fig. 7. Relationship between heterogeneity index of the pore size distributions obtained from two different presentations in the samples (Sample \#1-Sample \#6).

parameters that are calculated from the $D V / D \log d$ curve. However, $D V / D \log d$ curve has a larger $\alpha_{10}$ value. The heterogeneity index that is calculated from the $D V / D d$ curve is greater than the heterogeneity index from the $D V / D \log d$ curve. Fig. 7 compares the heterogeneity index estimated from these two different presentations where a linear correlation can be found between them. Collectively, the multifractal analysis results showed that heterogeneity of the pore size distributions calculated from the $D V / D d$ curve is more significant compared to the $D V / D \log d$ in the same sample.

The impact of rock compositions (mineral and organic part) on the heterogeneity index is further analyzed and discussed here. The partial linear least square regression method was employed which is found to be a suitable form of analysis of relationship that would exist between PSD heterogeneity and rock composition (Liu et al., 2017). The rock compositions including the minerals and the organic matter will be considered as the independent parameters while the heterogeneity index can be viewed as the dependent parameters. The results in Fig. 8 show that feldspar has the most significant effect on the heterogeneity index of both presentations. Carbonate has a negative effect on the heterogeneity index while the other constituent components would have a positive effect on PSD heterogeneity. To summarize, the effect of rock compositions on the heterogeneity index that is obtained from these two

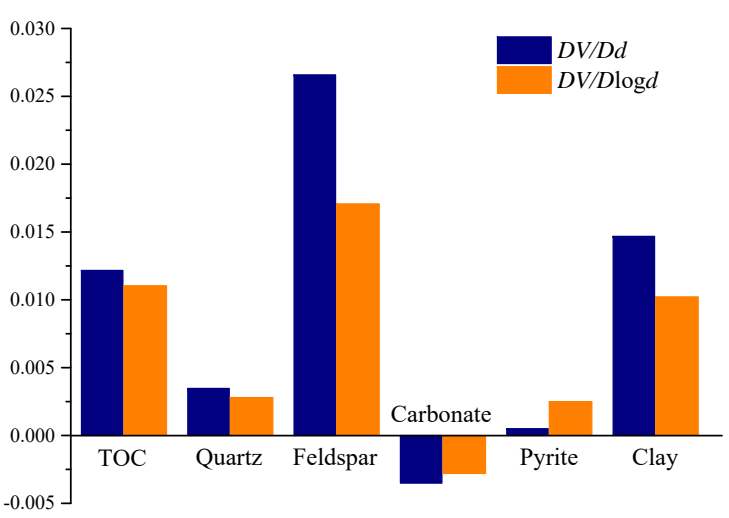

Fig. 8. The Influence of the minerals/TOC on the heterogeneity of pore size distributions on the two different presentations of the data.

presentations is similar while the intensity is quite different. $D V / D d$ curve for example, the effect that rock compositions have on the heterogeneity index based on their order of the intensity is: Feldspar $>$ clay $>$ organic matter, while in the $D V / D \log d$ curve, this order follows: Feldspar $>$ organic matter > clay.

\subsection{High pressure mercury intrusion}

Another important method to characterize pore structures of shale or any rock medium is high pressure mercury intrusion porosimetry. In this part, additional five samples (Sample \#7, $\# 8$, \#9, \#10, \#11) were collected and analyzed with MIP (Liu et al., 2019a). After being vacuum-dried in the oven at 70 ${ }^{\circ} \mathrm{C}$ for over 10 hours, the samples were put in the mercury porosimeter (Auto Pore IV 9,510, Micrometrics Instrument). Capillary pressure curves vs. the volume of mercury intruding sample pores were recorded as the injection pressure is increased from 0 up to 60,000 psia (413.68 MPa) (Liu et al., 2019a). The mineral compositions of these five samples are presented in Table 4, indicating that quartz and clay are the major components of these rock samples. All the samples are organic rich with TOC content more than $10 \mathrm{wt}$. \%.

The black curve in Fig. 9 represents the $D V$ curve and the blue curve denotes the $D V / D d$ curve data presentation. Both curves have noticeable unimodal characteristics. Alike the $\mathrm{N}_{2}$ adsorption data, where the major peak appears is different in each form of PSD data presentation. In this regard, the major peak of the $D V$ curve is found in larger pore radii in 


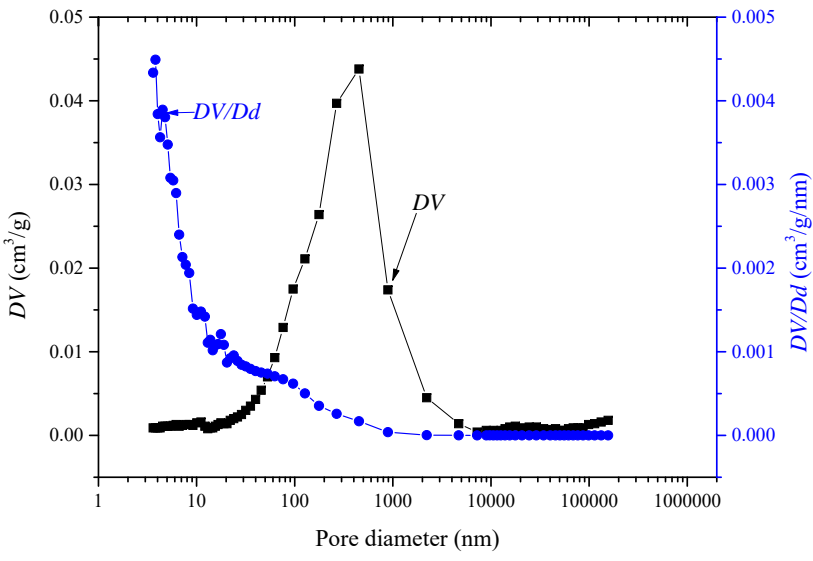

(a) Sample \#7

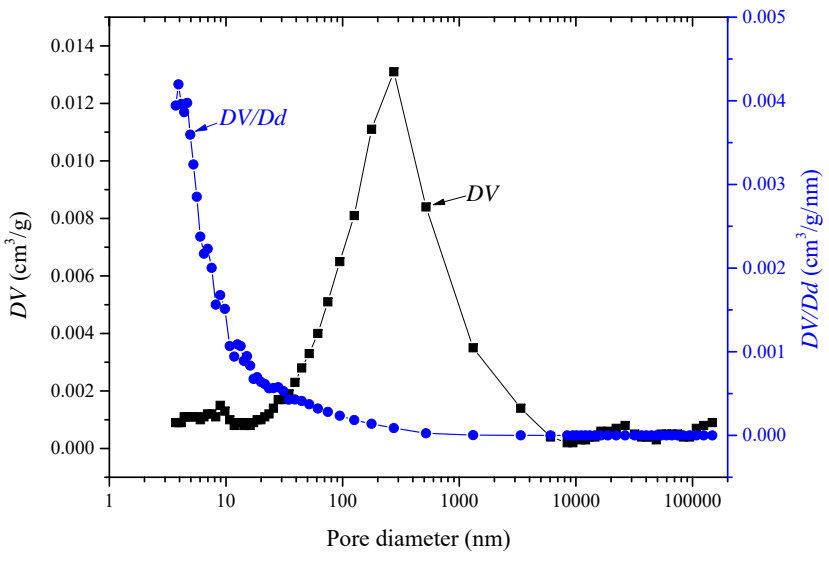

(b) Sample \#8

Fig. 9. Comparison of the $D V$ curve (black) and $D V / D d$ curve (blue) data presentation.

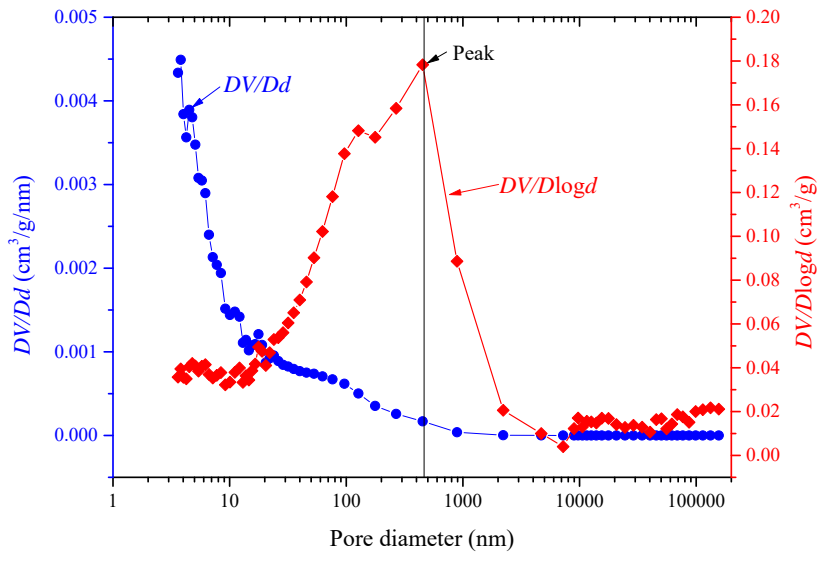

(a) Sample \#7

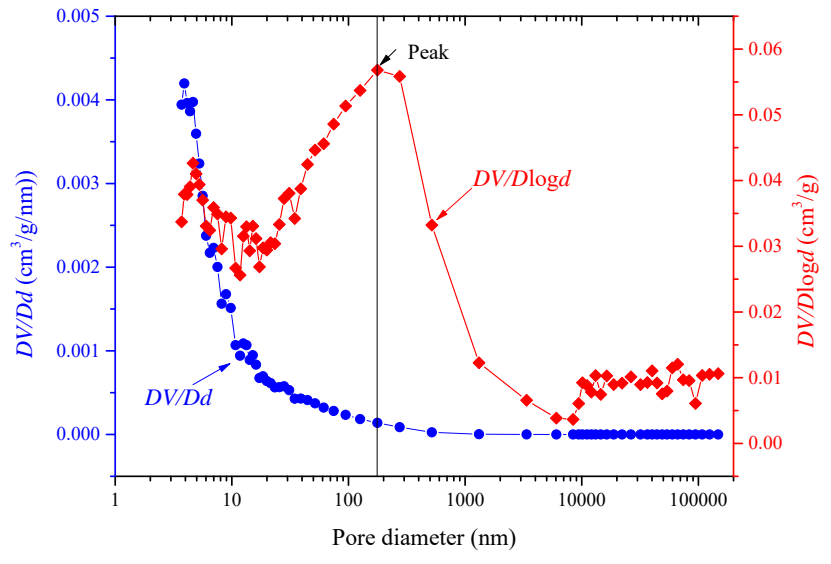

(b) Sample \#8

Fig. 10. Comparison of the $D V / D d$ curve (blue) and the $D V / D \log d$ curve (red) and existing major peaks.

comparison to the major peak of the $D V / D d$.

A better comparison of $D V / D d$ curve and the $D V / D \log d$ curve can be seen in Fig. 10 where major differences can be distinguished between the two curves. The blue curve $(D V / D d)$ shows the unimodal characteristics while the red curve $(D V / D \log d)$ from the same sample has a multimodal behavior where several peaks (pore diameters) exist in the $D V / D \log d$ curve that are missing in the $D V / D d$ curve. Taking Sample \#7 for example, the peak that is associated with the pore size diameter of around $500 \mathrm{~nm}$ exists in the $D V / D \log d$ curve but cannot be distinguished in the $D V / D d$ curve. Additionally, major peaks of the $D V / D \log d$ curve correspond to larger pore sizes than major peaks in $D V / D d$ curve.

Following the same analysis that was carried out for the $\mathrm{N}_{2}$ adsorption, the heterogeneity parameters of these two different presentations $(D V / D d$ and $D V / D \log d)$ were compared. The correlations between $\alpha_{q}$ and $q$ of Sample \#7 and Sample \#8 is displayed in Fig. 11. Considering both curves in the same sample, $\alpha_{0}>\alpha_{1}>\alpha_{2}$ explains that mercury intrusion has the multifractal characteristics in both form of presentation of the data. The comparison of the heterogeneity indices of the pore size distributions from these two different presentations is depicted in Fig. 12. The results show that heterogeneity index values that are obtained from the $D V / D d$ curve are generally larger than values that are obtained from the $D V / D \log d$ curve.

The impact that rock composition would have on the heterogeneity index from these two different presentations can be seen in Fig. 13. The results indicate that the impact of rock compositions on the heterogeneity index of these two different presentations are similar based on individual component. For example, organic matter has the most significant positive effect on the heterogeneity index while clay has a negative impact on the heterogeneity index that is calculated from these two different presentations. Furthermore, the magnitude of this impact from individual components on heterogeneity index of these two different presentations follows the same order.

\section{Recommendations}

The analysis that was laid out above demonstrate that diff- 


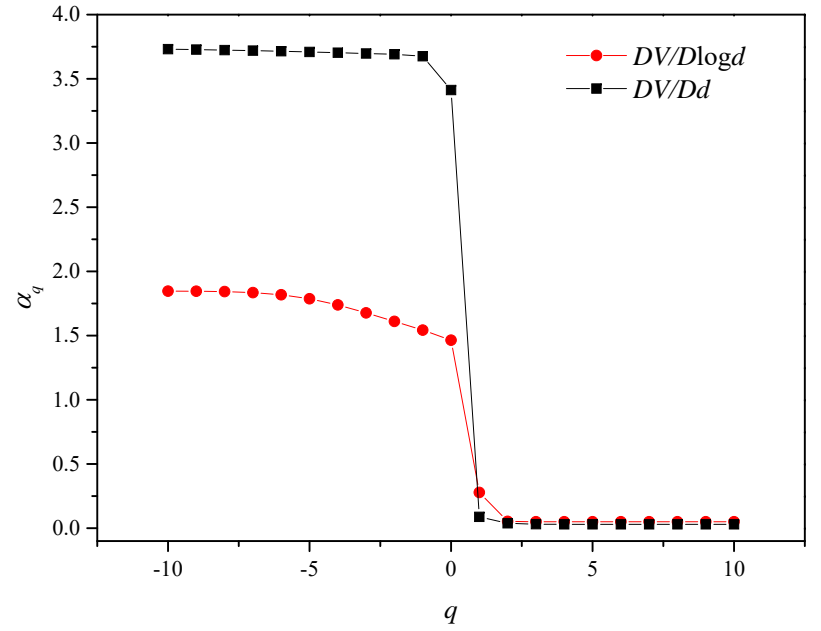

(a) Sample \#7

Fig. 11. The correlations between the $\alpha_{q}$ and $q$.

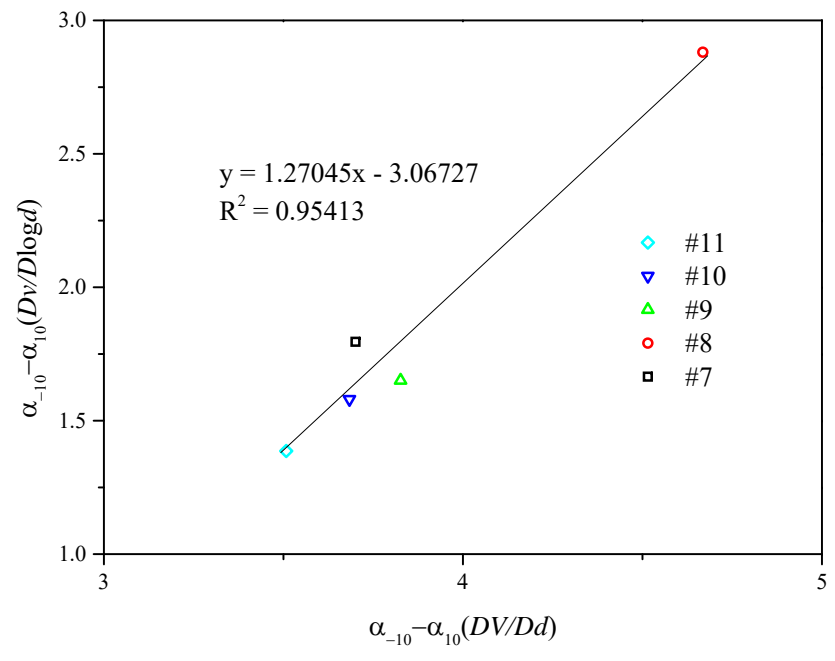

Fig. 12. Relationship between heterogeneity index of pore size distribution data from two different presentations (Sample \#7 to Sample \#11).

erent form of presentations of PSD data can lead to a different interpretation of the data such as the location of major peaks (pore size), peak intensity (pore frequency), the resulting heterogeneity and the impact of rock compositions on the heterogeneity index. Furthermore, the differential pore volume distribution is defined as the difference between two diameter boundaries over which the volume is determined. Considering this definition, the endpoints in the PSD intervals and the magnitude of their expression plays a major role in the results. The upper endpoint may be expressed in tens of nanometers $\left(\mathrm{N}_{2}\right.$ gas adsorption) or hundreds of micrometers (mercury intrusion) while the lower endpoint will be around less than 1 nanometer $\left(\mathrm{N}_{2}\right.$ adsorption) or few nanometers (mercury intrusion), respectively. Particularly in MIP for example, the

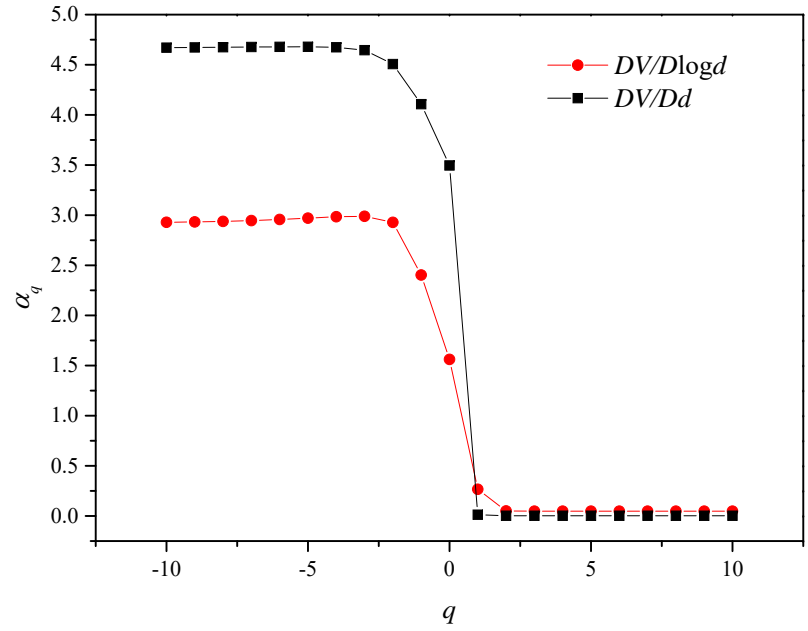

(b) Sample \#8

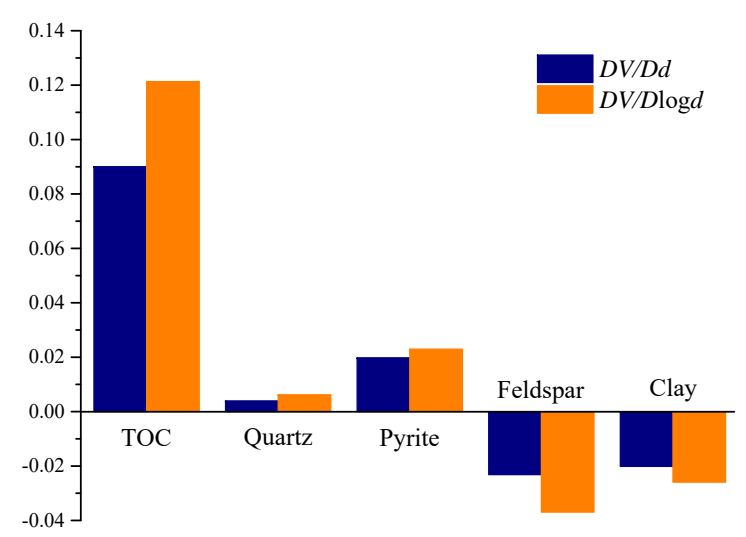

Fig. 13. The influence of individual minerals/TOC on the heterogeneity from the pore size distribution data from the two different presentations.

endpoints that are either expressed in micrometer or nanometer $[(50 \mu \mathrm{m}-100 \mu \mathrm{m})$ or $(5 \mathrm{~nm}-10 \mathrm{~nm})$, for example], the increments will represent equal distances along the $\mathrm{x}$-axis if they are plotted in the logarithmic scale. Considering the intrusion volume $(1 \mathrm{~mL} / \mathrm{g})$ over these two amount of interval increments (50 $\mu \mathrm{m}, 5 \mathrm{~nm}$, respectively), the differential pore volume per diameter over these increments will be 0.02 and $200 \mathrm{~mL} / \mathrm{g} / \mu \mathrm{m}$, respectively. For the equal amount of pore volumes, the intensity of the $D V / D d$ at one interval $(100 \mu \mathrm{m}$ $50 \mu \mathrm{m})$ is only around $0.01 \%$ of the other interval $(10 \mathrm{~nm}-5$ $\mathrm{nm})$. This means, the presentation of the $D V / D d$ curve can signify the role that smaller pore diameter ranges would have on PSD data while suppressing the contribution from larger pore diameter intervals.

From mathematics point of view, the correlation between $D V / D \log d$ and $D V / D d$ is defined by the following equation (Meyer and Klobes, 1999):

$$
\frac{D V}{D \log r}=2.303 r \frac{D V}{D r}
$$


where $r$ is the pore radius.

It's found in Eq. (4), that the $2.303 r$ term is the weighting parameter for the $D V / D \log d$ curve which will magnify the impact of pores at larger radii. MIP intervals that were used above, for example, the log difference between the largest incremental interval $(\log (100 \mu \mathrm{m})-\log (50 \mu \mathrm{m}))$ will be 0.3 which is close to the log difference of the smallest incremental interval $(\log (0.01 \mu \mathrm{m})-\log (0.005 \mu \mathrm{m}))$ of pore diameters. If the intrusion volume $(1 \mathrm{~mL} / \mathrm{g})$ over these two interval increments was the same, then the intensity of the peaks for log differential pore volume distributions at both ends will be similar.

Overall, the $D V / D d$ will magnify the smaller pore ranges and suppress the larger pore ranges while the $D V / D \log d$ has an opposite effect. In shale samples, the $\mathrm{N}_{2}$ adsorption can measure the smaller pores ranges from 2-200 nm with the majority of the pores from $2-50 \mathrm{~nm}$ thus the $D V / D d$ curve is preferred which can better characterize PSD. On the contrary, in mercury intrusion, since the high pressure from the mercury intruding the pores can distort pore structures, especially in smaller ones due to the compressibility effects, one can conclude the smaller pore diameter ranges that are obtained from the mercury intrusion might not be accurate. Therefore, in order to suppress the effect from the contributing or occupied smaller pores, the $D V / D \log d$ curve is preferred for data presentation in MIP. In this study, the goal was to focus on the effect of the presentations on the PSD interpretations from two mainstream experimental methods, the $\mathrm{N}_{2}$ gas adsorption and MIP, which can characterize the pores with sizes larger than $2 \mathrm{~nm}$ combined. Based on the results from this study, the authors highly recommend investigating discrepancies in data interpretation from different presentation types on the small micropores with sizes less than $2 \mathrm{~nm}$ that are measured by low-pressure $\mathrm{CO}_{2}$ adsorption method since this pore size range are abundant in shales.

\section{Conclusions}

In order to understand how presenting pore size distribution data would affect the pore structure interpretation of shale rocks, three different main types of data presentations from $\mathrm{N}_{2}$ adsorption and high-pressure mercury intrusion on several shale samples from the Bakken were compared. The main conclusions of this study can be listed as:

1) The $D V$ curve depends largely on the experimental data points spacing/intervals.

2) In $\mathrm{N}_{2}$ adsorption, the deconvolution of the PSD curves showed that some peaks representing pore sizes around 10-40 nm exist in both $D V / D d$ and $D V / D \log d$ curves. The $D V / D d$ curve can provide us with more peaks in the smaller pore ranges compared to $D V / D \log d$. The heterogeneity index obtained from the $D V / D d$ curve is more significant than the heterogeneity index from the $D V / D \log d$ curve. Linear correlations exist between the heterogeneity indexes from these two different presentations.

3) Considering high-pressure mercury intrusion, the location and the intensity of the peaks from the $D V / D d$ and
$D V / D \log d$ representing various pore radii found to differ. The heterogeneity index from the $D V / D d$ curve was larger than the heterogeneity index from the $D V / D \log d$. The impact of rock compositions on the heterogeneity index of these two presentations was found to be similar.

4) $D V / D d$ will incite the presence of smaller pore ranges and suppress the existence of larger pores while the $D V / D \log d$ has an opposite effect. When analysing the $\mathrm{N}_{2}$ adsorption data, the $D V / D d$ presentation should be picked based on the results from this study, while for analysing of the mercury intrusion data, the $D V / D \log d$ presentation is superior based on the pore sizes that each method can detect.

\section{Acknowledgments}

The authors appreciate the support from China Scholarship Council (No. 201406450029). We'd like to also show our appreciation to ND Core Library, Jeff Bader the director and state geologist as well as Kent Holland library technician for providing us with the samples. Authors would like to also thank the reviewers and journal editor for constructive feedback and comments.

Open Access This article is distributed under the terms and conditions of the Creative Commons Attribution (CC BY-NC-ND) license, which permits unrestricted use, distribution, and reproduction in any medium, provided the original work is properly cited.

\section{References}

Bustin, R.M., Bustin, A.M., Cui, A., et al. Impact of shale properties on pore structure and storage characteristics. Paper SPE1 19892 Presented at SPE Shale Gas Production Conference, Texas, USA, 16-18 November, 2008.

Cao, T., Song, Z., Wang, S., et al. A comparative study of the specific surface area and pore structure of different shales and their kerogens. Sci. China Earth Sci. 2015, 58(4): 510-522.

Clarkson, C.R., Solano, N., Bustin, R.M., et al. Pore structure characterization of North American shale gas reservoirs using USANS/SANS, gas adsorption, and mercury intrusion. Fuel 2013, 103: 606-616.

Clarkson, C.R., Wood, J., Burgis, S.E., et al. Nanopore structure analysis and permeability predictions for a tight gas/shale reservoir using low-pressure adsorption and mercury intrusion techniques. Paper SPE155537 Presented at SPE Americas Unconventional Resources Conference, Pennsylvania, USA, 5-7 June, 2012.

Do, D.D., Do, H.D. Pore characterization of carbonaceous materials by DFT and GCMC simulations: A review. Adsorp. Sci. Technol. 2003, 21(5): 389-423.

Ferreiro, J.P., Miranda, J.G.V., Vzquez, E.V. Multifractal analysis of soil porosity based on mercury injection and nitrogen adsorption. Vadose Zone J. 2010, 9(2): 325-335.

Gao, Z., Hu, Q. Estimating permeability using median pore-throat radius obtained from mercury intrusion porosimetry. J. Geophys. Eng. 2013, 10(2): 025014.

Han, H., Cao, Y., Chen, S., et al. Influence of particle size on gas-adsorption experiments of shales: An example from a 
Longmaxi Shale sample from the Sichuan Basin, China. Fuel 2016, 186: 750-757.

Hazra, B., Wood, D.A., Vishal, V., et al. Pore-characteristics of distinct thermally mature shales: Influence of particle sizes on low pressure $\mathrm{CO}_{2}$ and $\mathrm{N}_{2}$ adsorption. Energy Fuels 2018, 32(8): 8175-8186.

Kuila, U. Measurement and interpretation of porosity and pore-size distribution in mudrocks: The hole story of shales. Colorado, Colorado School of Mines, 2013.

Kumar, S., Mendhe, V.A., Kamble, A.D., et al. Geochemical attributes, pore structures and fractal characteristics of Barakar shale deposits of Mand-Raigarh Basin, India. Mar. Pet. Geol. 2019, 103: 377-396.

Liu, K., Ostadhassan, M., Kong, L., et al. Multifractal characteristics of Longmaxi Shale pore structures by $\mathrm{N}_{2}$ adsorption: A model comparison. J. Pet. Sci. Eng. 2018, 168: 330-341.

Liu, K., Ostadhassan, M., Kong, L., et al. Fractal and multifractal characteristics of pore throats in the Bakken shale. Transp. Porous Media 2019a, 126(3): 579-598.

Liu, K., Ostadhassan, M., Sun, L. A comprehensive pore structure study of the Bakken Shale with SANS, $\mathrm{N}_{2}$ adsorption and mercury intrusion. Fuel 2019b, 245: 274285.

Liu, K., Ostadhassan, M., Zou, J., et al. Nanoscale pore structure characterization of the Bakken shale in the USA. Fuel 2017, 209: 567-578.

Liu, M., Mostaghimi, P. Characterisation of reactive transport in pore-scale correlated porous media. Chem. Eng. Sci. 2017, 173: 121-130.

Mastalerz, M., Schimmelmann, A., Drobniak, A., et al. Porosity of Devonian and Mississippian New Albany Shale across a maturation gradient: Insights from organic petrology, gas adsorption, and mercury intrusion. AAPG Bull. 2013, 97(10): 1621-1643.

Meyer, K., Klobes, P. Comparison between different presentations of pore size distribution in porous materials. Fresenius J. Anal. Chem. 1999, 363(2): 174-178.
Sorelli, L., Constantinides, G., Ulm, F.J., et al. The nanomechanical signature of ultra high performance concrete by statistical nanoindentation techniques. Cem. Concr. Res. 2008, 38(12): 1447-1456.

Tang, X., Jiang, Z., Li, Z., et al. The effect of the variation in material composition on the heterogeneous pore structure of high-maturity shale of the Silurian Longmaxi formation in the southeastern Sichuan Basin, China. J. Nat. Gas Sci. Eng. 2015, 23: 464-473.

Thommes, M., Kaneko, K., Neimark, A.V., et al. Physisorption of gases, with special reference to the evaluation of surface area and pore size distribution (IUPAC Technical Report). Pure Appl. Chem. 2015, 87(9-10): 1051-1069.

Ulm, F.J., Vandamme, M., Bobko, C., et al. Statistical indentation techniques for hydrated nanocomposites: Concrete, bone, and shale. J. Am. Ceram. Soc. 2007, 90(9): 2677-2692.

Wang, L., Fu, Y., Li, J., et al. Mineral and pore structure characteristics of gas shale in Longmaxi formation: A case study of Jiaoshiba gas field in the southern Sichuan Basin, China. Arab. J. Geosci. 2016, 9(19): 733.

Wang, M., Xie, W., Huang, K., et al. Fine characterization of lithofacies and pore network structure of continental shale: case study of the Shuinan Formation in the north Jiaolai Basin, China. J. Pet. Sci. Eng. 2019, 175: 948-960.

Washburn, E.W. The dynamics of capillary flow. Phys. Rev. 1921, 17(3): 273.

Yang, Y., Yao, J., Wang, C., et al. New pore space characterization method of shale matrix formation by considering organic and inorganic pores. J. Nat. Gas Sci. Eng. 2015, 27: 496-503.

Yuan, Y., Rezaee, R., Verrall, M. Pore characterization and clay bound water assessment in shale with a combination of NMR and low-pressure nitrogen gas adsorption. Int. J. Coal Geol. 2018, 194: 11-21.

Zou, J., Rezaee, R., Xie, Q., et al. Investigation of moisture effect on methane adsorption capacity of shale samples. Fuel 2018, 232: 323-332. 


\section{Appendix}

Deconvolution procedure (Ulm et al., 2007; Sorelli et al., 2008; Liu et al., 2017).

The pores in the samples can be divided into $J=1, n$ pore size groups with sufficient contrast in pore size distributions. The $J_{t h}$ pore group occupies a volume fraction $f_{J}$ of the total porosity. The theoretical probability density function (PDF) of the single phase, assuming to fit a normal distribution is defined as:

$$
P_{J}\left(x_{i}, U_{J}, S_{J}\right)=\frac{2}{\sqrt{2 \pi\left(S_{J}\right)^{2}}} \exp \left[\frac{-\left(x_{i}-U_{J}\right)^{2}}{2\left(S_{J}\right)^{2}}\right]
$$

where $U_{J}$ and $S_{J}$ are the mean value and the standard deviation of pore size distributions of the phase $J=1$ to $n$.

After that, we minimize the difference between the data from the weighted model-phase PDF and the experimental PDF Eqs. (A-2) and (A-3):

$$
\begin{gathered}
\min \left\{\sum_{i=1}^{m} \sum_{J}^{N}\left[\sum_{J}^{n} f_{J} P_{J}\left(x_{i}, U_{J}, S_{J}\right)-P_{x}\left(x_{i}\right)\right]^{2}\right\} \\
\sum_{J=1}^{n} f_{J}=1
\end{gathered}
$$

In the above equation, $P_{x}\left(x_{i}\right)$ is the measured value of the normalized frequency of the pore size $x_{i}$ and $m$ is the number of the intervals (bins).

To ensure that the pore size groups have sufficient contrast, the overlap of successive Gaussian curves representative of the two phases is constrained by the following criterion:

$$
U_{J}+S_{J}<U_{J+1}+S_{J+1}
$$

